\title{
Effectiveness of Hand Self-Shiatsu to Promote Sleep in Young People with Chronic Pain: a Case Series Design
}

Cary A. Brown, PhD, ${ }^{1}$ Annette Rivard, PhD, ${ }^{1}$ Kathy Reid, NP, ${ }^{3}$ Bruce Dick, PhD, ${ }^{4}$ Leisa Bellmore, MSc, ${ }^{5}$ Pei Qin, MSc, ${ }^{2}$ Vineet Prasad, MSc, ${ }^{2}$ Yuluan Wang, MSc ${ }^{2}$

'Department of Occupational Therapy, Faculty of Rehabilitation Medicine, University of Alberta, Edmonton, $A B,{ }^{2}$ Faculty of Rehabilitation Medicine, University of Alberta, Edmonton, AB, ${ }^{3}$ Stollery Children's Hospital,

Edmonton, Alberta, ${ }^{4}$ Department of Anesthesiology and Pain Medicine, University of Alberta, Edmonton, $A B$, ${ }^{5}$ Artists' Health Centre, Toronto Western Hospital, University Health Network, Toronto, ON, Canada

Background: Sleep problems exist for up to 30\% of young people, and increase in the case of those with chronic pain. Because exclusive pharmacological management of sleep problems for children with pain is contraindicated, the development of appropriate non-pharmacological sleep interventions is a significant, largely unmet, need.

Purpose: This study examined whether the application of a standardized hand self-shiatsu (HSS) intervention within a population of young people with chronic pain would be associated with improved objectively and subjectively measured sleep.

Setting: The Pain Management Clinic of the Stollery Children's Hospital Hospital, a large tertiary care centre in Edmonton, Alberta and the University of Alberta.

Research Design: Sixteen young adults, aged 17 to 27, were recruited for a case series study. The intervention involved participants self-applying a standardized hand shiatsu protocol. Participants wore an actigraph for one week at baseline before learning the HSS technique, and then at four- and eight-week follow-up. At the same measurement points they completed validated self-report measures of their sleep quality and daytime fatigue. Each participant also completed a sleep log to supplement the actigraphy data and to collect their general impressions of the HSS experience. Data were analyzed with SPSS 23 software, using Freidman's test for analysis of variance.

Results: The objective data did not support the hypothesis that this standardized HSS protocol improves objectively measured sleep. However, standardized self-report measures demonstrated statistically significant improvement in perceived sleep disturbance (chi-squared test $\left.\left[\chi^{2}\right]=8.034, p=.02\right)$, sleep-related impairment $\left(\chi^{2}=7.614, p=.02\right)$, and daytime fatigue as measured by the PROMIS Fatigue SF 8-a $\left(\chi^{2}=12.035, p=.002\right)$, and the Flinder's Fatigue Scale $\left(\chi^{2}=11.93, p=\right.$ .003). Qualitative sleep log information indicated wide-spread endorsement of HSS for the management of sleep difficulties.

Conclusion: Contrary to objective findings, self-report data support the technique of HSS to improve sleep. Participants' comments reflected an overall high level of acceptance and appreciation for the HSS technique. Results highlight the importance of expanding the theory and practice related to sleep measurement to better integrate the qualitative domain.

KEY WORDS: insomnia; young people; intervention; fatigue; pain; self-management; shiatsu

\section{INTRODUCTION}

The prevalence of chronic pain (CP) in young Canadians has been measured to be at rates of $4 \%$ for abdominal pain, $8 \%$ for headache pain, and $14-24 \%$ for back pain. (1) Chronic pain is resistant to intervention, and takes a negative toll on young people's physical, emotional, and cognitive development such that social development and academic achievement are overwhelmingly challenged. ${ }^{(2,3)}$ Additionally, young peoples' CP experiences can impact their 
families, including negatively influencing the emotional health and resilience of parents. ${ }^{(4)}$ More specifically, CP significantly influences sleep, one of the foundational elements for health and resiliency. The importance of addressing sleep problems, (including insomnia, shortened sleep duration, and perceptions that sleep was nonrestorative) in pain management programs for pediatric and young adult populations has been well-demonstrated.(5) Sleep problems, typically self-reported and enduring, are experienced by up to $30 \%$ of young people(6) and this prevalence increases for those with CP.(7)

Exclusive pharmacological management of sleep problems for young people with pain is contraindicated.(8) Cognitive behavioural therapy (CBT) techniques are considered the 'gold-standard' for both the treatment of pediatric chronic pain(7) and insomnia in young people. (9) However, CBT may not be acceptable, nor accessible, to everyone for a variety of reasons including location, cost, presence of a support network, self-image, and past experience.(10) Indeed, a review of CBT outcomes found an attrition rate of up to $25 \%$. (11) The identification of acceptable and accessible non-pharmacological sleep interventions (NPSI) for youth with CP is thus a significant unmet need. Shiatsu, which is based on traditional Chinese medicine theory proposed to work by restoring and balancing body energy and reducing stress, ${ }^{(12)}$ is an example of a NPSI that can be incorporated into sleep management programs. Namikoshi shiatsu, on which the hand self-shiatsu (HSS) technique in this study is based, isolates points based on anatomy and physiology. ${ }^{(13)}$ Shiatsu involves applying pressure with the fingertip to specific points aligned with bodily functions and health and, from the perspective of Western medicine, is believed to trigger physiological benefits to blood circulation, muscle tension, and the production of serotonin. (14) Three studies examining the effects of shiatsu have produced promising findings. ${ }^{(15-17)}$ However, those studies were primarily carried out with older adults, and third-parties provided the shiatsu intervention. Reliance on a third party to deliver an NPSI presents issues of access, frequency, cost, and practicality of use. ${ }^{(18)}$

There is evidence in the pain literature supporting patient self-management models and these are favoured by most intervention services. ${ }^{(19)}$ Self-management approaches help patients regain feelings of mastery and control.(20) Current brain functioning and attention research ${ }^{(21)}$ proposes that the level of sustained on-task concentration, such as that required to apply HSS, can reduce, and even prevent, the risk of an individual simultaneously attending to other thoughts. ${ }^{(18,22)}$ Actively self-administering shiatsu may thus preclude concentrating on intrusive thoughts and emotions, which trigger the production of sleep-inhibiting, pain-promoting, stress-related neurotransmitters and hormones such as cortisol. (23) Cognition-based interventions to decrease insomniacs' rumination have been effective, (24) and the active concentration required to carry out HSS aligns with this approach.

Two previous studies examining HSS for improving sleep suggested that it may be beneficial for chronic pain patients with diagnosed musculoskeletal conditions, as well as for young athletes with sportrelated concussion, who also self-reported sleep difficulties. ${ }^{(18,22)}$ These studies proposed that, because HSS involves concentrating on a motor activity as opposed to ruminating and worrying on personal thoughts and feelings, it is more appealing to persons who find the interpersonal reflection components of cognitive therapies unacceptable. Indeed, participants in both studies reported a high degree of acceptance and satisfaction with the intervention. Hand self-shiatsu aligns with the evidence-based principles of selfmanagement and can be practised within the patient's unique biopsychosocial and environmental context. (22) It is unavoidably portable and accessible, and can be performed either sitting or lying down, in the dark before bed and when patients wake up during the night.

Given the need to increase self-management options, the endorsement of NPSI for young people living with chronic pain who also experience sleep deficiency, and the promising trends in both objective and self-report measures identified in past HSS pilot studies, ${ }^{(18,22)}$ this study's goal was two-fold. First, to examine whether a standardized HSS protocol improved select sleep dimensions as quantified by a wrist-worn sleep monitor (actigraph) and to examine if the HSS protocol improved sleep quality and daytime fatigue as measured by self-report instruments. We also sought to explore participants' attitudes about, and adherence to, HSS. In 
an effort to detect potential confounding variables, participants' beliefs about sleep, holistic health, and the scientific validity of complementary/alternative medicine were also measured.

\section{METHODS}

\section{Study Design}

A case series design (clinical series in which there is known exposure) with participants acting as their own controls was used. This method is well suited for hypothesis generation and preliminary testing of novel interventions where a strong evidence-base does not yet exist.(25) The study protocol was approved by the University of Alberta Health Ethics Board, No. Pro00070452_REN2. Written consent was obtained from all study participants and from parents if under 18 years of age. All training occurred either in a university classroom or at a public meeting space of the participant's choice (e.g., library or coffee shop).

\section{Participants}

Participants were recruited from the Chronic Pain Program at the Stollery Children's Hosptial through individual letters of invitation to persons attending the program, and through the University of Alberta student email platform, "Mailman". Participants were accepted into the study if they were 16 to 28 years of age and selfreported difficulty with sleep. Exclusion criteria included: inability to communicate in English, an active arthritic condition involving the hand or with unhealed hand injuries, using sleep medication with prescription changed during the eightweek study.

\section{Intervention}

The same standardized HSS protocol as that used by Brown et al.(22) and Qin et al.(18) was implemented (available at https:// cbotlabs.wixsite.com/handselfshiatsu). This involved participants self-applying pressure with the pads of the index finger or thumb for a count of three to specific points on the dorsal and volar surfaces of the opposite hand and fingers (four rows of three points between the long bones on the dorsum of the hand, three points on the centre of the palm, three points on the front, back, and sides of each thumb, and four points on the front, back, and sides of each finger). Participants applied HSS once they were in bed, with the lights out, and ready for sleep. The sequence was applied twice, taking approximately 10 to 15 minutes to complete. Each participant was trained to apply HSS in a one-to-one session following one week of baseline sleep actigraphy and self-report data collection. Printed HSS materials (available on the above website) were provided to participants to facilitate intervention fidelity. Participants were contacted by telephone in the week after HSS training to clarify questions about the technique.

\section{Measurement}

Data collection consisted of various tools to measure both objective and selfreported dimensions of sleep and day-time fatigue. Potential confounding factors were also measured.

\section{Objective Outcomes}

Real time sleep was assessed using the wrist-worn Actisleep monitor manufactured by Actigraph [https://actigraphcorp. $\mathrm{com} /]$. Data are uploaded and analyzed using proprietary software. Actigraphy is considered to be a reliable and valid assessment tool and is widely used in sleep research. (26) Participants wore the device for seven days at baseline (BL) prior to intervention, and at four (FU1) and eight (FU2) weeks following HSS training. Six sleep dimensions were retained for analysis: sleep onset latency, waking after sleep onset, average time awake, average number of awakenings, sleep efficiency (ratio of time asleep to time spent in bed), and total sleep time. Lower scores indicate improvement in sleep onset latency, waking after sleep onset, average time awake, average number of awakenings, while higher scores reflect improvement in sleep efficiency and total sleep time.

\section{Sleep Log}

Participants completed a sleep log each morning and before bed for seven days, at baseline (BL) (prior to intervention), and for one week at FU1 and FU2. Sleep logs are commonly used to record individuals' sleep-related information, and a study with a similar young adult population demonstrated a sensitivity of $87.93 \%$ 
and a specificity of $96.51 \%$ in relation to objective actigraphy data.(27) In this study, the sleep log collected bed and wake up times for entry into the Actisleep software for analysis of downloaded monitor data, and the number of awakenings at night. Participants also indicated whether they had practised HSS before bed and during the night.

\section{Self-report Measures}

Sleep quality measurements (including sleep disturbance, sleep-related impairment, and daytime fatigue data) were taken at BL, and again at FU1 and FU2. Specifically, participants completed the Pittsburgh Sleep Quality Index (PSQI), a widely used, psychometrically strong, self-report measure that assesses sleep quality and disturbances over the previous month. (28) Nineteen individual items generate seven component scores: subjective sleep quality, sleep latency and duration, habitual (usual) sleep efficiency, sleep disturbances, use of sleep medications, and daytime dysfunction. The sum of the scores for the seven components yields a global score with a possible range of 0 to 21 points, with higher scores signifying poorer sleep. A global score of 5 or greater indicates poor sleep quality.

Three scales developed by the Patient Reported Outcomes Measurement Information System (PROMIS) program were utilized.(29) These measures have been shown to possess strong psychometric properties. For each scale, raw mean scores are converted to standardized T- scores, with a mean of 50 and a standard deviation of 10. The measures selected for this study each consist of eight items, measured on a five-point scale, and ask participants to rate the past seven days. The Sleep Disturbance Short Form 8a assesses selfreported perceptions of sleep quality, sleep depth, and restoration associated with sleep, including difficulties with getting to sleep or staying asleep, and perceptions of the adequacy and satisfaction with sleep. The Sleep-Related Impairment-Short Form 8a focuses on perceptions of alertness, sleepiness, and tiredness during usual waking hours, and the perceived functional impairments during wakefulness associated with sleep problems and impaired alertness. The Fatigue-Short Form 8 a was used to measure self-reported experience of fatigue (frequency, duration, and intensity) and the impact of fatigue on physical, mental, and social activities, on a five-point scale. For all three scales, higher scores indicate worse outcomes-that is to say, greater sleep disturbance, greater sleep-related impairment and greater fatigue. The 7-item Flinders Fatigue Scale, (30) a valid and reliable instrument that measures daytime fatigue experienced over the previous two weeks, was also used. A clear description of the term "fatigue" is provided in the initial instructions to the scale, and six questions are presented in Likert format, with responses ranging from 1 (not at all) to 4 (extremely or entirely). The items address themes of how problematic fatigue is to the individual, the consequences of fatigue, its frequency and severity, and insomnia patients' perception of fatigue's association with sleep. Total fatigue is calculated as the sum of all individual items, with higher scores indicating greater fatigue.

\section{Confounding Variables}

In order to determine whether other factors that could change sleep behaviour (such as educational or self-directed information seeking) occurred simultaneously during the HSS protocol, participants completed the Sleep Beliefs Scale ${ }^{(31)}$ at BL and FU2. This scale contains 20 statements describing select behaviours and asks participants whether each has a positive, negative, or no effect on the quality and quantity of sleep in general. Examples of belief statements include "going to bed and waking up at the same hour', and 'getting up when it is difficult to fall asleep'. Participants also completed the Holistic Complementary and Alternative Medicine Questionnaire,(32) an 11-item questionnaire that measures attitudes about holistic health and the scientific validity of complementary and alternative medicine. This measure, with demonstrated test-retest reliability and internal validity, contains six items about attitudes towards complementary and alternative medicine, and five items about holistic health beliefs.

\section{Statistical Analysis}

Data were analyzed using IBM SPSS 23.0 software. Friedman's Test was used to analyze the variance between BL, FU1, and FU2 measurements, with a Bonferronicorrected alpha-level of 0.025 to account for two comparisons-a) between Baseline and follow-up 1, and b) between follow-up 
1 and follow-up 2. Wilcoxon's test was used to examine potential changes in the confounding variable scores with alpha-level of 0.05 .

\section{RESULTS}

Eighteen participants agreed to participate in the study, of which 16 completed all phases of the study. One person dropped out without providing a reason and a second was excluded from the data analysis when it was learned that he was working night shifts at the time of the study. Seventy-eight percent $(n=14)$ of participants were female. The mean age was 21.56 , ranging from 17-27 years. With respect to pain duration, $65 \%(n=11)$ of participants reported experiencing chronic pain for between $\mathrm{O}$ to 5 years, and 35\% $(n=6)$ reported experiencing pain for 5 to 10 years. Type of pain reported was classified according to the ICD-11 and included back pain and fibromyalgia, headache and orofacial pain, visceral pain, and musculoskeletal pain.(33) Forty-seven percent $[n=8]$ of participants reported chronic primary pain, $24 \%(n=4)$ reported chronic headache and orofacial pain, $18 \%$ $(n=3)$ reported musculoskeletal pain, and $12 \%[n=2]$ experienced visceral pain.

\section{Actigraphy}

As depicted in Table 1, Friedman's test revealed no significant differences in any of

TABLE 1. Actigraphy Results

\begin{tabular}{lccccc}
\hline & \multicolumn{5}{c}{ Mean Ranks $^{a}$} \\
\cline { 2 - 4 } & BL & FU1 & FU2 & $\chi^{2}$ & $P$ \\
\hline SOL & 2.28 & 1.78 & 1.94 & 2.23 & .33 \\
WaSO & 2.00 & 1.81 & 2.19 & 1.13 & .57 \\
ATA & 1.69 & 2.16 & 2.16 & 2.42 & .30 \\
\#A & 2.19 & 1.88 & 1.94 & .875 & .65 \\
SE & 1.88 & 2.19 & 1.94 & .875 & .65 \\
TST & 1.94 & 2.19 & 1.88 & .875 & .65 \\
\hline
\end{tabular}

aUsing Friedman's Test; threshold for statistical significance was set at .025 .

$\mathrm{BL}=$ baseline; FU1 = follow-up 1, FU2 = follow-up 2;

$\mathrm{SOL}=$ Sleep onset latency; $\mathrm{WaSO}=$ average awakenings after sleep onset; ATA = average time awake; \#A = number of awakenings; SE = sleep efficiency; TST = total sleep time. the six actigraphy sleep dimensions across the three measurement time points.

\section{Sleep Log}

All sixteen participants reported using HSS before bed at FU1 and FU2, albeit not necessarily for each of the seven nights. Fifty per cent and $44 \%$ reported also using HSS if they woke during the night at FU1 and FU2, respectively. Table 2 depicts participants' adherence to the HSS protocol in greater detail.

The self-report sleep logs revealed a potentially concerning lack of established sleep routine in many of the participants, with some demonstrating a variation of more than 2 hours between earliest and latest bedtime recorded. Eighty-eight per cent of participants reported an average variation greater than 2 hours in their bedtimes over the three measurement periods, with one participant exhibiting a mean range variation of 7.5 hours. The sleep logs also revealed high variation in the duration of sleep periods from night to night for a number of participants.

\section{Self-reported Measures}

Results of all self-reported measures were analyzed using Freidman's test to identify changes at BL through FU1 and FU2. Results are displayed in Table 3. Scores obtained from the PSQI followed a normal distribution and ranged from 2

TABLE 2. Frequency of HSS Protocol Completions During Data Collection Periods

\begin{tabular}{lcc}
\hline & $\begin{array}{c}\text { Follow Up 7: } \\
n(\%)\end{array}$ & $\begin{array}{c}\text { Follow Up 2: } \\
n(\%)\end{array}$ \\
\cline { 2 - 3 } \# of nights & \multicolumn{2}{c}{ HSS Before Bed } \\
\hline $6-7$ nights & $9(56 \%)$ & $10(63 \%)$ \\
$4-5$ nights & $7(44 \%)$ & $3(18.7 \%)$ \\
$1-3$ nights & 0 & $3(18.7 \%)$ \\
Total & 16 & 16 \\
& HSS During the Night \\
$6-7$ nights & $2(13 \%)$ & $2(13 \%)$ \\
$4-5$ nights & $1(6 \%)$ & 0 \\
$1-3$ nights & $5(31 \%)$ & $5(31 \%)$ \\
Total & 8 & 7 \\
\hline
\end{tabular}


TABLE 3. Self-Report Measures

\begin{tabular}{|c|c|c|c|c|c|}
\hline & \multicolumn{3}{|c|}{ Mean Ranks ${ }^{a}$} & \multirow[b]{2}{*}{$\chi^{2}$} & \multirow[b]{2}{*}{$P$} \\
\hline & $B L$ & FU1 & FU2 & & \\
\hline PSQI & 10.68 & 9.18 & 8.18 & 6.746 & .034 \\
\hline Sleep Disturbance & 2.41 & 2.13 & 1.47 & 8.034 & .018 \\
\hline $\begin{array}{l}\text { Sleep-regulated } \\
\text { impairment }\end{array}$ & 2.41 & 2.09 & 1.50 & 7.614 & .022 \\
\hline $\begin{array}{l}\text { Fatigue Short } \\
\text { Form }\end{array}$ & 2.56 & 2.03 & 1.41 & 12.035 & .002 \\
\hline Finders Fatigue & 2.63 & 1.94 & 1.44 & 11.93 & .003 \\
\hline
\end{tabular}

aUsing Freidman Test; ( $N=16$; $d f=2)$; threshold for statistical significance was set at .025.

PSQI = Pittsburgh Sleep Quality Index; BL = baseline; FU1 = follow-up 1, FU2 = follow-up 2.

to 20. Analysis indicated that the changes in scores across the three measurement points were not statistically significant. Analysis of the Sleep Disturbance Short Form 8-a scores indicated a statistically significant change across the three time points $\left(\chi^{2}=8.034, p=.02\right)$. Analysis of the Sleep-Related Impairment Short Form 8-a scores indicated a significant change $\left(\chi^{2}\right.$ $=7.614, p=.02$ ), with mean ranks showing a steady decline over the three measurement periods. A steady decline in mean rank scores on the Fatigue-8a short form over the three measured periods was observed. The change across the three measurements was statistically significant $\left(\chi^{2}=12.035, p=.002\right)$. Analysis also demonstrated a consistent decrease in mean rank scores on the Flinders Fatigue Scale over the three measurement periods that was statistically significant $\left(\chi^{2}=11.93\right.$, $p=.003)$.

\section{Potential Confounding Variables}

There was no change in scores on the Sleep Beliefs Scale potentially indicating that participants did not learn 'new' information during the study that pertained to conditions impacting on sleep (see Table 4). The HCAMQ results also indicated no significant change in scores between BL and FU2. Its two subscales, 'complementary and alternative medicine' and 'holistic health beliefs', were analyzed separately. Again, there were no changes in these scores from BL to FU 2.
TABLE 4. Results for the Confounding Variables ${ }^{a}$

\begin{tabular}{lcccc}
\hline & $\begin{array}{c}\text { Sleep } \\
\text { Beliefs } \\
\text { Scale }\end{array}$ & $\begin{array}{c}\text { CAM } \\
\text { (HCAMHQ } \\
\text { sub-scale) }\end{array}$ & $\begin{array}{c}\text { HH } \\
\text { (HCAMHQ } \\
\text { subcale) }\end{array}$ & $\begin{array}{c}\text { HCAMHQ } \\
\text { Total } \\
\text { Score }\end{array}$ \\
\hline $\begin{array}{l}\text { Z (rank } \\
\text { score) } \\
\text { Sig }\end{array}$ & 1.683 & 1.027 & 1.065 & .821 \\
\hline
\end{tabular}

aUsing Wilcoxon test; threshold for statistical significance was set at .05.

HCAMQ = Holistic Complementary and Alternative Medicine Questionnaire; CAM = Complementary and Alternative Medicine; $\mathrm{HH}=$ holistic health.

\section{DISCUSSION}

The primary objective of this work was to examine whether a standardized hand selfshiatsu intervention with a population of young adults experiencing poor sleep and who self-identified as living with chronic pain would result in improved objectively and subjectively measured sleep.

While there was no statistically significant change in objective measures of sleep, results from the self-report measures demonstrated statistically significant improvement in perceived sleep disturbance, sleep-related impairment, and daytime fatigue. This pattern of improvement in self-report sleep measures without measurable change in actigraph is consistent with the findings in two previous pilot studies of HSS. ${ }^{(18,22)}$ This discrepancy in objective VS. self-reported measures reinforces the notion that sleep, given its complex bio-psycho-social nature, eludes unidimensional measurement. Indeed, several other sleep intervention studies have demonstrated a similar pattern of positive findings from self-report measures without corresponding actigraphy evidence.(34-36)

The relevance of applying both actigraphy and self-report measures to capture sleep-related outcomes is particularly important for this study's population given the growing body of evidence demonstrating that persons with chronic pain, which can only be measured subjectively, are more sensitive to the effects of poor sleep. (36) Pain patients may have keener perceptions relative to their psychological and physiological states than what an actigraph can accurately measure. As such, self-reports may be required to capture a 
more nuanced picture of sleep. The need for a multiple-domain approach to sleep assessment is underscored by a 2018 review of sleep assessment tools which concluded that a synergy between subjective and objective measures is paramount to sleep detection. (37)

With respect to the lack of established sleep routine, given that these are selfreports collected over short seven-day sleep periods, it is premature to draw any conclusions. However, other researchers have found a statistically significant relationship between day-to-day variability in sleep duration and poor subjective wellbeing including lower satisfaction with life and higher depressive symptoms, anxious symptoms, loss of interest, and anxious arousal scores. ${ }^{(38)}$ This is highly relevant given the prevalence of mental health issues in the young adult chronic pain patient population. (39)

There were some additional narrative data collected in the sleep logs relative to possible confounding factors, such as pre-bedtime activity and sleep environment conditions. This will provide useful information for the development of future, more stringent, studies. For example, 75\% of participants reported using electronic devices during the hour before bed. As there is compelling evidence that this activity delays sleep onset, ${ }^{(40)}$ it is reasonable to suspect that electronic use may have interfered with the effectiveness of HSS. As our goal was to examine a pragmatic intervention that would be acceptable to young people with pain, requiring young people with many complex social challenges to refrain from electronic use pre-bedtime was not integrated into the intervention protocol.

At the termination of the study, 10 participants responded to the query as to whether they would recommend HSS to others. Nine stated that they would do so, while one felt more time was needed for the trial. Respondents provided clear endorsement for HSS as depicted in the following quotes: "I found it helped me fall asleep faster and helped me have a more restful sleep"; "I found it had a great sedative effect and was relaxing"; "I find it calms me down and helps regulate my thoughts better"; and "even though the HSS did not quantitatively improve or worsen my sleep quality, I did feel some improvements by being more energized when waking up". Interestingly, one respondent endorsed the sleep log itself as a worthwhile endeavour for improving sleep:

"I found that even on days when I didn't do the actual hand shiatsu, simply being mindful of the sleep log and filling it out to indicate I missed a day still allowed me to be more self-aware of other aspects of my daily routine that impacts sleep .... The sleep log was the one aspect of this experience that proved to be very helpful."

In summary, these findings align with previous HSS pilot studies involving adult chronic pain patients(22) and young athletes with sport related concussion, ${ }^{(18)}$ that suggest that an additional or alternative mechanism of change, aligning with current research in attention and concentration, might be present. Further controlled studies involving electroencephalography to examine neural activity during HSS, strategies to improve HSS protocol adherence, and the management of potential confounding factors (such as electronic screen use and activity patterns), are needed. Future research should also compare HSS to other forms of self-administered body work (such as general hand self-massage and acupressure) and outcomes of studies involving HSS in combination with other forms of sleep intervention (such as cognitive behavioural therapy).

It is important to note that the Namikoshi shiatsu protocol for this study was developed by an experienced Western-trained shiatsu practitioner and, as such, may not reflect the related cultural elements of a practitioner trained in Japan where the founder of this approach, Tokujiro Namikoshi, developed his practice.(13) There are several other study limitations; the sample size was small, there was no control group, adherence to the HSS technique between follow-up periods was not monitored, electronic screen time and other sleepinhibiting activities pre-bedtime were not controlled for, and HSS technique fidelity was not monitored. Additionally, as the study was conducted during the academic year, it is possible that sleep schedules varied for a number of the participants in relation to the demands of school work. However, we did attempt to mitigate this by scheduling follow-up periods during regular class times, and avoiding holiday and exam periods where more sporadic sleep 
patterns might be expected. Though adherence to the HSS protocol was good during the two follow-up measurement periods, we cannot ascertain the frequency of HSS use in-between these periods as those data were not collected. A final limitation was that the last follow-up at eight weeks may have been too early to identify whether the HSS practice had become a sustained habit.

These findings did not support the hypothesis that a standardized HSS protocol used by young people with chronic pain at bedtime and during night time awakenings would improve sleep efficiency as quantified by a wrist-worn sleep monitor (actigraph). However, sleep is a complex experience in which objective and subjective outcomes are important. Participants' demonstration of significant self-reported improvement in sleep disturbance, sleeprelated impairment, and daytime fatigue, coupled with their wide-spread endorsement of HSS for the management of sleep difficulties, are findings of note.

These results highlight that HSS holds potential for improved sleep outcomes and underscore the importance of expanding theory and practice related to sleep measurement to better integrate the qualitative domain and refine the manner in which sleep interventions are evaluated.

\section{ACKNOWLEDGMENTS}

This research has been funded by the generous support of the Women and Children's Health Research Institute (WCHRI).

\section{CONFLICT OF INTEREST NOTIFICATION}

The authors declare there are no conflicts of interest.

\section{COPYRIGHT}

Published under the CreativeCommons Attribution-NonCommercial-NoDerivs 3.0 License.

\section{REFERENCES}

1. King S, Chambers CT, Huguet A, MacNevin RC, McGrath PJ, Parker L, et al. The epidemiology of chronic pain in children and adolescents revisited: a systematic review. Pain. 2011;152(12):2729-2738
2. Palermo TM, Valrie CR, Karlson CW. Family and parent influences on pediatric chronic pain: a developmental perspective. Am Psychol. 2014;9(2):142-152.

3. Hunfeld JA, Perquin CW, Duivenvoorden $\mathrm{HJ}$, Hazebroek-Kampschreur AA, Passchier J, van Suijlekom-Smit LW, et al. Chronic pain and its impact on quality of life in adolescents and their families. J Pediatr Psychol. 2001;26(3):145-153.

4. Eccleston C, Crombez G, Scotford A, Clinch J, Connell $\mathrm{H}$. Adolescent chronic pain: patterns and predictors of emotional distress in adolescents with chronic pain and their parents. Pain. 2004;108(3):221-229.

5. Valrie CR, Bromberg MH, Palermo T, Schanberg LE. A systematic review of sleep in pediatric pain populations. J Dev Behav Pediatr. 2013;34(2):120-128.

6. Chaput JP, Janssen I. Sleep duration estimates of Canadian children and adolescents. J Sleep Res. 2016;25(5):541-548.

7. Palermo TM, Toliver-Sokol M, Fonareva I, Koh JL. Objective and subjective assessment of sleep in adolescents with chronic pain compared to healthy adolescents. Clin J Pain. 2007;23(9):812-820.

8. Carter BD, Threlkeld BM. Psychosocial perspectives in the treatment of pediatric chronic pain. Pediatr Rheumatol. 2012;10(1):15.

9. Mindell JA, Meltzer LJ. Behavioural sleep disorders in children and adolescents. Ann Acad Med Singapore. 2008;37(8):722-728.

10. Cheung JM, Bartlett DJ, Armour CL, Saini B. Treating insomnia: a review of patient perceptions toward treatment. Behav Sleep Med. 2016;14(3): 235-266.

11. Fernandez E, Salem D, Swift JK, Ramtahal N. Meta-analysis of dropout from cognitive behavioral therapy: magnitude, timing, and moderators. Consult Clin Psychol. 2015;83(6):1108-1122.

12. Makoto A. Shiatsu for women's health. Aust Nurs Midwifery J. 2013;21(3):51.

13. Namikoshi, T. Shiatsu Therapy: Theory and Practice. Tokyo: Japan Publications Inc.; 1974.

14. Robinson N, Lorenc A, Liao X. The evidence for shiatsu: a systematic review of shiatsu and acupressure. BMC Complement Alt Med. 2011;11(1):88.

15. Yuan SL, Berssaneti AA, Marquez AP. Effects of shiatsu in the management of fibromyalgia symptoms: a controlled pilot study. J Manipulative Physiol Ther. 2013;36(7):436-443.

16. Long AF, Mackay HC. The effects of shiatsu: findings from a two-country exploratory study. J Altern Complement Med. 2003;9(4):539-547.

17. Long AF. The effectiveness of shiatsu from a crossEuropean prospective observational study. J Altern Complement Med. 2008;14(8):921-930.

18. Qin P, Dick B, Leung A, Brown CA Effectiveness of hand self-shiatsu to improve sleep following sport-related concussion in young athletes: a proof-of-concept study. J Integrative Med. 2019;17:24-29. 
19. Reid MC, Papaleontiou M, Ong A, Breckman R, Wethington E, Pillemer K. Self-management strategies to reduce pain and improve function among older adults in community settings: a review of the evidence. Pain Med. 2008;9(4):409-424.

20. Moseley GL. Evidence for a direct relationship between cognitive and physical change during an education intervention in people with chronic low back pain. Eur J Pain. 2004;8(1):39-45.

21. Styles E. The Psychology of Attention. New York: Psychology Press; 2006.

22. Brown CA, Bostick G, Bellmore L, Kumanayaka D. Hand self-shiatsu for sleep problems in persons with chronic pain: a pilot study. $J$ of Integrative Med. 2014;12(2):94-101.

23. Hannibal KE, Bishop MD. Chronic stress, cortisol dysfunction, and pain: a psychoneuroendocrine rationale for stress management in pain rehabilitation. Phys Ther. 2014;94(12):1816-1825.

24. Harvey AG, Sharpley AL, Ree MJ, Stinson K, Clark DM. An open trial of cognitive therapy for chronic insomnia. Behav Res Ther. 2007;45(10):2491-2501.

25. Kooistra B, Dijkman B, Einhorn TA, Bhandari M. How to design a good case series. J Bone Joint Surg Am. 2009;91(Suppl. 3):21-26.

26. Zollman FS, Cyborski C, Duraski. Actigraphy for assessment of sleep in traumatic brain injury: case series, review of the literature and proposed criteria for use. Brain Injury. 2010;24(5):748-754.

27. Usui A, Ishizuka Y, Obinata I, Okado T, Fukuzawa $\mathrm{H}$, Kanba S. Validity of sleep log compared with actigraphic sleep-wake state II. Psychiatry Clin Neurosci.1999;53(2):183-184.

28. Buysse DJ, Reynolds CF 3rd, Monk TH, Berman SR, Kupfer DJ. The Pittsburgh Sleep Quality Index: a new instrument for psychiatric practice and research. Psychiatry Res. 1989;28(2):193-213.

29. Cella D, Young S, Rothrock N, Gershon R, Cook K, Reeve B, et al. The Patient-Reported Outcomes Measurement Information System (PROMIS): progress of an NIH Roadmap cooperative group during its first two years. Med Care. 2007;45(5 Suppl 1):S3-S11.

30. Gradisar M, Lack L, Richards H, Harris J, Gallasch J, Boundy $M$, et al. The Flinders Fatigue Scale: preliminary psychometric properties and clinical sensitivity of a new scale for measuring daytime fatigue associated with insomnia. J Clin Sleep Med. 2007;3(7):722-728.
31. Adan ANA, Fabbri M, Natale V, Prat G. Sleep Beliefs Scale (SBS) and circadian typology. J Sleep Res. 2006;15(2):125-132.

32. Hyland ME, Lewith GT, Westoby C. Developing a measure of attitudes: the holistic complementary and alternative medicine questionnaire. Complement Ther Med. 2003;11:33-38.

33. Treede RD, Rief W, Barke A, Aziz Q, Bennett MI, Benoliel R, et al. A classification of chronic pain for ICD-11. Pain. 2015;156(6):1003-1007.

34. Harvey AG, Tang NK. (Mis)perception of sleep in insomnia: a puzzle and a resolution. Psychol Bull. 2012;138(1):77.

35. Fernandez-Mendoza J, Calhoun SL, Bixler EO, Karataraki M, Liao D, Vela-Bueno A, et al. Sleep misperception and chronic insomnia in the general population: the role of objective sleep duration and psychological profiles. Psychosom Med. 2011;73(1):88.

36. O'Donnell D, Silva EJ, Muench M, Ronda JM, Wang W, Duffy JF. Comparison of subjective and objective assessments of sleep in healthy older subjects without sleep complaints. J Sleep Res. 2009;18(2):254-263.

37. Ibáñez V, Silva J, Cauli O. A survey on sleep assessment methods. PeerJ, 2018;6: e4849.

38. Jank R, Gallee A, Boeckle M, Fiegl S, Pieh C. Chronic pain and sleep disorders in primary care. Pain Res Treat. 2017; Article ID 9081802.

39. Lemola S, Ledermann T, Friedman EM. Variability of sleep duration is related to subjective sleep quality and subjective well-being: an actigraphy study. PLOS ONE. 2013;8(8):e71292.

40. Noel M, Groenewald CB, Beals-Erickson S, Gebert JT, Palermo TM. Chronic pain in adolescence and internalizing mental health disorders: a nationally representative study. Pain. 2016;157(6):1333-1338.

Corresponding author: Cary A. Brown, PhD, 2-64 Corbett Hall, Department of Occupational Therapy, Faculty of Rehabilitation Medicine, University of Alberta, Edmonton, AB, Canada T6G 2G4

E-mail: cary.brown@ualberta.ca 